\title{
Approximation Algorithms for Minimum-Width Annuli and Shells*
}

\author{
P. K. Agarwal, ${ }^{1}$ B. Aronov, ${ }^{2}$ S. Har-Peled,${ }^{3, \dagger}$ and M. Sharir ${ }^{3,4}$ \\ ${ }^{1}$ Center for Geometric Computing, Department of Computer Science, \\ Box 90129, Duke University, \\ Durham, NC 27708-0129, USA \\ pankaj@cs.duke.edu \\ ${ }^{2}$ Department of Computer and Information Science, Polytechnic University, \\ Brooklyn, NY 11201-3840, USA \\ aronov@ziggy.poly.edu \\ ${ }^{3}$ School of Mathematical Sciences, Tel Aviv University, \\ Tel Aviv 69978, Israel \\ sharir@math.tau.ac.il \\ ${ }^{4}$ Courant Institute of Mathematical Sciences, New York University, \\ New York, NY 10012, USA
}

\begin{abstract}
Let $S$ be a set of $n$ points in $\mathbb{R}^{d}$. The "roundness" of $S$ can be measured by computing the width $\omega^{*}=\omega^{*}(S)$ of the thinnest spherical shell (or annulus in $\mathbb{R}^{2}$ ) that contains $S$. This paper contains two main results related to computing an approximation of $\omega^{*}$ : (i) For $d=2$, we can compute in $O(n \log n)$ time an annulus containing $S$ whose width is at most $2 \omega^{*}(S)$. We extend this algorithm, so that, for any given parameter $\varepsilon>0$, an annulus containing $S$ whose width is at most $(1+\varepsilon) \omega^{*}$ is computed in time
\end{abstract}

\footnotetext{
* Work by P.A. was supported by Army Research Office MURI Grant DAAH04-96-1-0013, by a Sloan fellowship, by NSF Grants EIA-9870724 and CCR-9732787, by an NYI award, and by a grant from the U.S.-Israeli Binational Science Foundation. Work by B.A. was supported by a Sloan Research Fellowship and by a grant from the U.S.-Israeli Binational Science Foundation. Work by M.S. was supported by NSF Grants CCR-97-32101, CCR-94-24398, by grants from the U.S.-Israeli Binational Science Foundation, the G.I.F., the German-Israeli Foundation for Scientific Research and Development, and the ESPRIT IV LTR Project No. 21957 (CGAL), and by the Hermann Minkowski-MINERVA Center for Geometry at Tel Aviv University. Part of the work by P.A. and B.A. on the paper was done when they visited Tel Aviv University in May 1998. A preliminary version of the paper appeared as: P. K. Agarwal, B. Aronov, S. Har-Peled, and M. Sharir, Approximation and exact algorithms for minimum-width annuli and shells, Proc. 15th Annu. ACM Sympos. Comput. Geom., 1999, pp. 380-389.

$\dagger$ Current address: Center for Geometric Computing, Department of Computer Science, Box 90129, Duke University, Durham, NC 27708-0129, USA. sariel@cs.duke.edu.
} 
$O\left(n \log n+n / \varepsilon^{2}\right)$. (ii) For $d \geq 3$, given a parameter $\varepsilon>0$, we can compute a shell containing $S$ of width at most $(1+\varepsilon) \omega^{*}$ either in time $O\left(\left(n / \varepsilon^{d}\right) \log \left(\Delta / \omega^{*} \varepsilon\right)\right)$ or in time $O\left(\left(n / \varepsilon^{d-2}\right)(\log n+1 / \varepsilon) \log \left(\Delta / \omega^{*} \varepsilon\right)\right)$, where $\Delta$ is the diameter of $S$.

\section{Introduction}

Let $S$ be a set of $n$ points in $\mathbb{R}^{d}$. The roundness of $S$ can be measured by approximating $S$ with a sphere $\Gamma$ so that the maximum distance between a point of $S$ and $\Gamma$ is minimized, i.e., by computing

$$
\min _{c \in \mathbb{R}^{d}, r \in \mathbb{R}} \max _{p \in S}|d(p, c)-r|
$$

For $c \in \mathbb{R}^{d}$ and for $r, R \in \mathbb{R}$ with $0 \leq r \leq R$, we define the spherical shell (shell, for short, and, in the plane, annulus) $\mathcal{A}(c, r, R)$ to be the closed region lying between the two concentric spheres of radii $r$ and $R$ centered at $c$. The width of $\mathcal{A}(c, r, R)$ is $R-r$. The problem of measuring the roundness of $S$ is equivalent to computing a shell, $\mathcal{A}^{*}(S)$, of the smallest width that contains $S$. See Fig. 1.

The main motivation for computing a minimum-width shell or annulus comes from metrology. For example, the circularity of a two-dimensional object $O$ in the plane is measured by sampling a set $S$ of points on the surface of $O$ (e.g., using coordinate measurement machines) and computing the width of the thinnest shell containing $S$ [14]. Motivated by this and other applications, the problem of computing $\mathcal{A}^{*}(S)$ in the plane has been studied extensively [2]-[5], [11], [12], [15], [18], [20], [22]-[27], [29]. Ebara et al. [11] noticed that in the planar case the center of $\mathcal{A}^{*}(S)$ is a vertex of the overlay of the nearest- and farthest-neighbor Voronoi diagrams of $S$. This property was later refined and extended in [24] and [27]. These observations immediately lead to an $O\left(n^{2}\right)$-time algorithm for computing $\mathcal{A}^{*}(S)$ in the plane. Subquadratic algorithms were later developed in [2]-[4]. The asymptotically fastest known randomized algorithm, by Agarwal and Sharir [3], computes $\mathcal{A}^{*}(S)$ in expected time $O\left(n^{3 / 2+\varepsilon}\right)$, for any $\varepsilon>0$. Since the subquadratic algorithms are rather complicated, simpler and faster algorithms have been developed for various special cases [8], [9], [18], [28]. Mehlhorn et al. [20] and Kumar and Sivakumar [17] have studied this problem under the probing model in which the set $S$ of sample points is chosen adaptively; see the original papers for details.

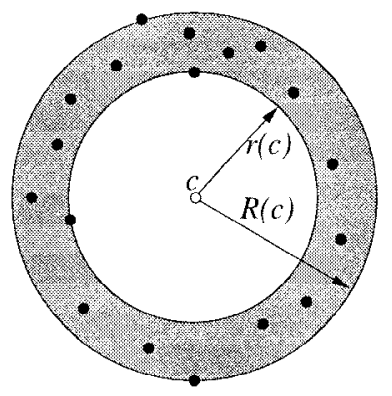

Fig. 1. The annulus $\mathcal{A}^{*}(S)$. 
Very little was known about computing $\mathcal{A}^{*}(S)$ efficiently in higher dimensions. Extending the observation by Ebara et al. [11] to $\mathbb{R}^{3}$, it can be shown that the center of $\mathcal{A}^{*}(S)$ is the intersection point of an edge of the nearest-neighbor Voronoi diagram of $S$ with a face of the farthest-neighbor Voronoi diagram of $S$, or vice versa. Using this fact, $\mathcal{A}^{*}(S)$ can be computed in $O\left(n^{3} \log n\right)$ time [9]. This idea can also be extended to higher dimensions. Very recently, Chan [7] pointed out that the three-dimensional problem can be solved exactly in a very simple manner in time $O\left(n^{2}\right)$; in fact his observation gives a procedure in all dimensions. (Chan's result improves an exact algorithm presented in the preliminary version of this paper [1].)

Since the exact version of the problem, even in the plane, does not have so far any simple efficient solutions, it is of interest to develop such algorithms that compute an enclosing annulus or shell whose width is a good approximation to the optimal width in near linear time. Very little has been done so far in this direction, and previous work [9], [15] solved the problem under additional assumptions concerning the input data.

This paper contains two main results that involve approximation algorithms for the minimum-width enclosing annulus or shell:

(i) For $d \geq 2$, given a parameter $\varepsilon>0$, we present simple algorithms that run either in time $O\left(\left(n / \varepsilon^{d}\right) \log \left(\Delta / \omega^{*} \varepsilon\right)\right)$ or in $O\left(\left(n / \varepsilon^{d-2}\right)(\log n+1 / \varepsilon) \log \left(\Delta / \omega^{*} \varepsilon\right)\right)$ for computing a shell that contains $S$ and whose width is at most $(1+\varepsilon) \omega^{*}$, where $\omega^{*}$ is the width of $\mathcal{A}^{*}(S)$ and $\Delta=\operatorname{diam}(S)$ is the diameter of $S$ (Section 3). If the middle radius (i.e., average of the inner and outer radii) of $\mathcal{A}^{*}(S)$ is at most $U \cdot \operatorname{diam}(S)$, then the running time of the algorithms are $O\left(\left(n / \varepsilon^{d}\right) \log (U+1)\right)$ and $O\left(\left(n / \varepsilon^{d-2}\right)(\log n+1 / \varepsilon) \log (U+1)\right)$, respectively. In most practical situations, $U$ is a constant. For example, if the input points span an angle of at least $\theta$ with respect to the center of $\mathcal{A}^{*}(S), U=O(1 / \theta)$.

A main idea used in the algorithms is the observation that, in the plane, the minimumarea annulus containing $S$ can be used to approximate $\mathcal{A}^{*}(S)$, and while this approximation might not always be good, it can at least be computed in linear time using linear programming. We refine this idea and extend it to higher dimensions to achieve the bounds stated above.

(ii) We derive simpler, faster algorithms for $d=2$. We first describe in Section 4.1 a very simple $O(n \log n)$-time algorithm for computing an annulus that contains $S$ and whose width is at most twice that of $\mathcal{A}^{*}(S)$. Duncan et al. [9] had described an approximation algorithm under some assumptions on the distribution of input points. No general near-linear-time algorithm with constant-factor approximation was previously known.

We then combine this algorithm with the previous one to obtain a $(1+\varepsilon)$-approximation algorithm. Given a parameter $\varepsilon>0$, we compute in $O\left(n \log n+n / \varepsilon^{2}\right)$ time an annulus that contains $S$ whose width is at most $(1+\varepsilon) \omega^{*}$, where $\omega^{*}$ is the width of $\mathcal{A}^{*}(S)$ (Section 4.2).

More recently, Chan obtained several new approximation results for the minimumwidth annulus/shell problem, using fairly simple techniques; refer to [7] for details.

\section{Geometric Preliminaries}

Let $S$ be a set of $n$ points in $\mathbb{R}^{d}$. For a point $p \in \mathbb{R}^{d}$, let $\mathrm{r}(p)$ (resp. $\mathrm{R}(p)$ ) denote the distance between $p$ and its nearest (resp. farthest) neighbor in $S . \mathcal{A}(p, \mathrm{r}(p), \mathrm{R}(p))$ is 


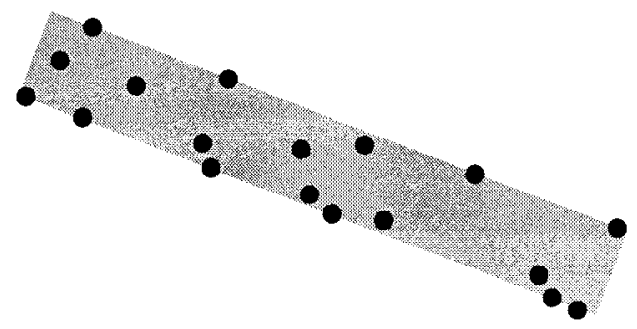

Fig. 2. The minimum-width annulus is realized by a center at infinity.

the shell of smallest width that is centered at $p$ and contains $S$, which we also denote by $\mathcal{A}(p)$. In what follows, unless we consider the problem specifically in the plane, we use the term "shell" to refer to a spherical shell in dimension higher than two and to an annulus in two dimensions. Set

$$
\omega(p)=\mathrm{R}(p)-\mathrm{r}(p) \quad \text { and } \quad r_{\text {mid }}(p)=\frac{\mathrm{R}(p)+\mathrm{r}(p)}{2} .
$$

We put $\omega^{*}=\omega^{*}(S)=\inf _{p \in \mathbb{R}^{d}} \omega(p)$ and denote by $\mathcal{A}^{*}=\mathcal{A}^{*}(S)$ a shell of width $\omega^{*}$ containing $S$. Note that the optimum value $\omega^{*}$ may not be attained by any finite point, in which case $\mathcal{A}^{*}(S)$ is a slab enclosed between two parallel hyperplanes, and $\omega^{*}(S)$ is then the standard width of $S$. See Fig. 2 for an illustration of this case. The following lemma states two simple but useful properties of $r_{\text {mid }}(p)$.

Lemma 2.1. Let $S$ be a finite set of points in $\mathbb{R}^{d}$. For any $p, q \in \mathbb{R}^{d}$, we have the following:

(i) $r_{\text {mid }}(p) \geq \mathrm{R}(p) / 2 \geq \operatorname{diam}(S) / 4$.

(ii) $\left|r_{\text {mid }}(p)-r_{\text {mid }}(q)\right| \leq d(p, q) \leq r_{\text {mid }}(p)+r_{\text {mid }}(q)$.

Proof. (i) is trivial to prove. To show (ii), use the inequalities

$$
\mathrm{r}(p) \leq d(p, q)+\mathrm{r}(q), \quad \mathrm{R}(p) \leq d(p, q)+\mathrm{R}(q), \quad d(p, q) \leq \mathrm{r}(p)+\mathrm{R}(q),
$$

whose proofs are straightforward.

Let $\operatorname{Vor}_{\mathrm{N}}(S)$ (resp. $\operatorname{Vor}_{\mathrm{F}}(S)$ ) denote the nearest-neighbor (resp. farthest-neighbor) Voronoi diagram of $S$. For $d=2$, let $\operatorname{Vor}_{\mathrm{N}}(S, \ell)$ denote the nearest-neighbor Voronoi diagram of $S$ restricted to a line $\ell$. That is, $\operatorname{Vor}_{N}(S, \ell)$ is the partition of $\ell$ into maximal intervals so that the same point of $S$ is closest to all points within each interval. The vertices of $\operatorname{Vor}_{\mathrm{N}}(S, \ell)$ are the intersection points of $\ell$ with the edges of $\operatorname{Vor}_{\mathrm{N}}(S)$. We can obviously compute $\operatorname{Vor}_{\mathrm{N}}(S, \ell)$ in $O(n \log n)$ time by first computing the entire $\operatorname{Vor}_{\mathrm{N}}(S)$ and then intersecting $\ell$ with it. However, $\operatorname{Vor}_{\mathrm{N}}(S, \ell)$ can be computed directly, in $O(n \log n)$ time, using a considerably simpler algorithm; see, e.g., [21]. We define $\operatorname{Vor}_{\mathrm{F}}(S, \ell)$ analogously; it can also be computed either directly in $O(n \log n)$ time or in $O(n)$ time after having computed $\operatorname{Vor}_{\mathrm{F}}(S)$. 


\section{An Approximation Algorithm in Any Dimension}

Let $S$ be a set of $n$ points in $\mathbb{R}^{d}$; we assume that $d$ is a small constant. Set $\Delta=\operatorname{diam}(S)$. We first describe an approximation algorithm for computing the thinnest shell $\mathcal{A}(p)$ containing $S$ with the constraint that

$$
r_{\text {mid }}(p)=\frac{\mathrm{r}(p)+\mathrm{R}(p)}{2} \leq U \cdot \Delta
$$

for some given positive parameter $U \in \mathbb{R}$. Let $\mathcal{A}^{*}(S, U)$ denote this constrained minimumwidth shell, and let $\omega^{*}(S, U)$ denote the width of $\mathcal{A}^{*}(S, U)$. Computing $\mathcal{A}^{*}(S, U)$ can be formulated as the following optimization problem in the $d+2$ variables $x_{1}, x_{2}, \ldots, x_{d}, r, R$ :

$$
\begin{array}{ll}
\text { minimize } & R-r \\
\text { subject to } & r \leq\left(\sum_{i=1}^{d}\left(x_{i}-p_{i}\right)^{2}\right)^{1 / 2} \leq R, \quad \forall p=\left(p_{1}, \ldots, p_{d}\right) \in S, \\
& r+R \leq 2 U \Delta .
\end{array}
$$

Let $C$ be a $d$-dimensional hyper-rectangle of the form $\prod_{i=1}^{d}\left[\alpha_{i}, \beta_{i}\right]$. We define another constrained shell $\mathcal{E}(S, C)$ (which becomes, when $d=2$, the minimum-area annulus containing $S$ with center constrained to lie in $C$ ), in the same variables, as follows:

$$
\begin{array}{lll}
\operatorname{minimize} & R^{2}-r^{2} \\
\text { subject to } & r \leq\left(\sum_{i=1}^{d}\left(x_{i}-p_{i}\right)^{2}\right)^{1 / 2} \leq R, \quad \forall p=\left(p_{1}, \ldots, p_{d}\right) \in S, \\
& \alpha_{i} \leq x_{i} \leq \beta_{i}, & 1 \leq i \leq d .
\end{array}
$$

If we substitute $\Sigma$ for $R^{2}-\sum_{i=1}^{d} x_{i}^{2}$ and $\sigma$ for $r^{2}-\sum_{i=1}^{d} x_{i}^{2}$, then $\Sigma-\sigma=R^{2}-r^{2}$, and we can restate the optimization problem defining $\mathcal{E}(S, C)$ as

$$
\begin{array}{lll}
\operatorname{minimize} & \Sigma-\sigma \\
\text { subject to } & \sigma \leq-\sum_{i=1}^{d} 2 p_{i} x_{i}+\sum_{i=1}^{d} p_{i}^{2} \leq \Sigma, & \forall p=\left(p_{1}, \ldots, p_{d}\right) \in S, \\
& \alpha_{i} \leq x_{i} \leq \beta_{i}, & 1 \leq i \leq d .
\end{array}
$$

This is, however, an instance of linear programming with $d+2$ variables, and can be solved in $O(n)$ time [10], [19], provided $d$ is a constant. Let $\hat{\omega}(S, C)$ denote the width of $\mathcal{E}(S, C)$.

We now describe our approximation algorithm. Let $C(p, s)$ be the $d$-dimensional axis-parallel cube of side length $s$ and centered at $p$. The approximation algorithm is depicted in Fig. 3.

Lemma 3.1. APPROX_Shell $(S, U, \varepsilon)$ returns a shell whose width is at most $(1+$ $\varepsilon) \omega^{*}(S, U)$. 
Algorithm APPROX_SHELL $(S, U, \varepsilon)$

1. Compute $\mathcal{A}_{0}=\mathcal{E}\left(S, \mathbb{R}^{d}\right)$. If $\hat{\omega}\left(S, \mathbb{R}^{d}\right)=0$, then return $\mathcal{E}\left(S, \mathbb{R}^{d}\right)$.

2. Pick a point $o \in S$ and set $\mathfrak{C}=C(o,(2 U+2) \Delta)$.

3. Partition $\mathfrak{C}$ into a collection $\mathcal{C}=\left\{C_{1}, \ldots, C_{k}\right\}$ of axis-parallel cubes so that, for all points $p, q$ inside the same cube $C_{i}, r_{\text {mid }}(p) \leq(1+\varepsilon) r_{\text {mid }}(q)$.

4. For each $C_{i} \in \mathcal{C}$, compute $\mathcal{A}_{i}=\mathcal{E}\left(S, C_{i}\right)$.

5. Return the thinnest shell among $\mathcal{A}_{0}, \mathcal{A}_{1}, \ldots, \mathcal{A}_{k}$.

Fig. 3. The $\varepsilon$-approximation algorithm APPROX_SHELL.

Proof. If $\hat{\omega}\left(S, \mathbb{R}^{d}\right)=0$, then the statement is obvious. Otherwise, let $p$ be the center of $\mathcal{A}^{*}(S, U)$. Since $r_{\text {mid }}(o) \leq \mathrm{R}(o) \leq \Delta$ and $r_{\text {mid }}(p) \leq U \Delta$, we have, by Lemma 2.1(ii), that $p \in \mathfrak{C}$. Let $C_{i}$ be the cube containing $p$. Let $q \in C_{i}$ be the center of $\mathcal{E}\left(S, C_{i}\right)$. Then

$$
\mathrm{R}^{2}(q)-\mathrm{r}^{2}(q) \leq \mathrm{R}^{2}(p)-\mathrm{r}^{2}(p) \quad \text { or } \quad r_{\text {mid }}(q) \omega(q) \leq r_{\text {mid }}(p) \omega(p) .
$$

Equivalently,

$$
\omega(q) \leq \frac{r_{\text {mid }}(p)}{r_{\text {mid }}(q)} \omega(p) \leq(1+\varepsilon) \omega^{*}(S, U)
$$

We now describe how to construct a partition $\mathcal{C}$ of $\mathfrak{C}$. A similar construction is given in [16].

Lemma 3.2. Let $U, \varepsilon$ be two positive numbers. Then $\mathfrak{C}=C(o,(2 U+2) \Delta)$ can be partitioned into a set $\mathcal{C}$ of $O\left((1 / \varepsilon)^{d} \log (U+1)\right)$ cubes so that $r_{\text {mid }}(p) \leq(1+\varepsilon) r_{\text {mid }}(q)$ for all $p, q$ in the same cube of the partition. This tiling can be computed in $O(n+$ $\left.(1 / \varepsilon)^{d} \log (U+1)\right)$ time.

Proof. Compute a real number $\mu$ such that $\Delta / 2 \leq \mu \leq \Delta$. (See [13] for a simple $O(n)$ algorithm for approximating the diameter to within a factor of $\sqrt{3}$ in any dimension. Alternatively, fix any $p \in S$ and take $\mu=\mathrm{R}(p) \geq \Delta / 2$, by Lemma 2.1(i).)

Set $m=\left\lceil\log _{2}(U+1)\right\rceil-1$. For $i=1, \ldots, m$, we define

$$
B_{0}=C(o, 4 \mu), \quad B_{i}=C\left(o, 2^{i+2} \mu\right) \backslash C\left(o, 2^{i+1} \mu\right) .
$$

We can tile $B_{0}$ by $O\left(1 / \varepsilon^{d}\right)$ axis-parallel cubes having side length $r_{0}=\mu \varepsilon /(4 \sqrt{d})$. Let $C$ be a cube in this tiling. For $p, q \in C$, we have, by Lemma 2.1,

$$
r_{\text {mid }}(p) \leq r_{\text {mid }}(q)+d(p, q) \leq r_{\text {mid }}(q)+\frac{\mu \varepsilon}{4} \leq(1+\varepsilon) r_{\text {mid }}(q),
$$

since $r_{\text {mid }}(q) \geq \Delta / 4 \geq \mu / 4$.

Let $r_{i}=2^{i} \mu \varepsilon / \sqrt{d}$, for $i=1, \ldots, m$. $B_{i}$ can be tiled by

$$
O\left(\left(\frac{2^{i+2} \mu}{r_{i}}\right)^{d}\right)=O\left(\left(\frac{2^{i+2} \mu}{2^{i} \mu \varepsilon / \sqrt{d}}\right)^{d}\right)=O\left(\frac{1}{\varepsilon^{d}}\right)
$$

axis-parallel cubes with side length $r_{i}$, for $i=1, \ldots, m$. 


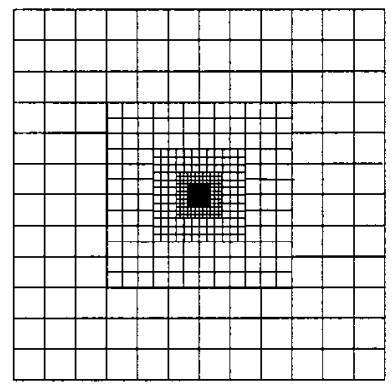

Fig. 4. Tiling of $\mathfrak{C}$.

Let $C$ be a cube in this tiling of $B_{i}$, and let $p, q$ be two points in $C$. Using Lemma 2.1(ii) and the fact that $r_{\text {mid }}(o) \leq \Delta \leq 2 \mu$, we have

$$
r_{\text {mid }}(q) \geq d(q, o)-r_{\text {mid }}(o) \geq 2^{i+1} \mu-2 \mu \geq 2^{i} \mu .
$$

We also have

$$
\begin{aligned}
r_{\text {mid }}(p) & \leq r_{\text {mid }}(q)+d(q, p) \leq r_{\text {mid }}(q)+\sqrt{d} r_{i} \\
& =r_{\text {mid }}(q)+2^{i} \mu \varepsilon \leq r_{\text {mid }}(q)(1+\varepsilon) .
\end{aligned}
$$

See Fig. 4 for an illustration of the resulting tiling. This completes the proof of the lemma, since $B_{m}$ contains $\mathfrak{C}$ and the total number of cubes is $O\left(\left(1 / \varepsilon^{d}\right) \log (U+1)\right)$. The bound on the running time of this construction is obvious.

Theorem 3.3. Let $S$ be a set of $n$ points in $\mathbb{R}^{d}, \varepsilon>0$, and $U>0$. One can compute a shell $\mathcal{A} \supset S$ whose width is at most $(1+\varepsilon) \omega^{*}(S, U)$ either in time $O\left(\left(n / \varepsilon^{d}\right) \log (U+1)\right)$ or in time

$$
O\left(\frac{n}{\varepsilon^{d-2}}\left(\log n+\frac{1}{\varepsilon}\right) \log (U+1)\right)
$$

Proof. The first bound on the running time is a consequence of the preceding discussion: We spend $O(n)$ time on each of the $O\left(\left(1 / \varepsilon^{d}\right) \log (U+1)\right)$ cubes of $\mathcal{C}$. The second bound follows by observing that the execution of the algorithm APPROX_SHELL can be interpreted as follows: We compute a sequence of cubes $\mathcal{C}_{1}, \ldots, \mathcal{C}_{m}$, where $m=O(\log (U+1))$. Each such cube is decomposed into $O\left(1 / \varepsilon^{d}\right)$ subcubes using an appropriate uniform grid. For each subcube $C$ we obtain $\mathcal{E}(S, C)$ as a solution of an appropriate linear-programming problem.

Let $\mathcal{C}_{i}$ be such a cube, and let $V=\left\{C_{1}, \ldots, C_{\mu}\right\}$ be the resulting decomposition of $\mathcal{C}_{i}$ into subcubes. The linear-programming instances on each $C_{j}$ are almost identical except for the $2 d$ inequalities restricting the solution to lie inside $C_{j}$. This implies that, with the possible exception of one subcube, the solutions to all those linear-programming instances must lie on the boundaries of the respective cubes $C_{1}, \ldots, C_{\mu}$. Moreover, the solution of at most one instance of the linear programming that does lie in the interior of its cube can be computed directly, by solving a single linear-programming instance, 
without restricting the location of the solution to any subcube (i.e., by dropping the inequalities $\alpha_{i} \leq x_{i} \leq \beta_{i}$ ).

In particular, we conclude that we can reduce the $d$-dimensional problem to a $(d-1)$ dimensional problem, as follows:

- Solve the unrestricted version of the linear programming (i.e., compute the global "minimum area" shell).

- For each axis-parallel $(d-1)$-dimensional hyperplane $H$ of the grid defining the decomposition $V$, find recursively a $(1+\varepsilon)$-approximate shell containing $S$ whose center is constrained to lie on $H \cap \mathcal{C}_{i}$. There are $O(d / \varepsilon)$ such hyperplanes.

- Return the shell of minimum width among all those generated by the algorithm.

The recursion bottoms out at $d=2$, where we proceed as follows. Let $H$ be our two-dimensional plane. We can compute in $O(n \log n)$ time the maps induced on $H$ by the $d$-dimensional nearest- and furthest-neighbor Voronoi diagrams of $S$ (these maps are called power diagrams [6], they have linear complexity, and they can be computed in $O(n \log n)$ time). Our target is to minimize the difference between the squared distances to the farthest and nearest neighbors of a point on $H$. We note that we can compute this minimum along a line $\ell$ in $O(n)$ time, by performing a walk through the overlay of those two diagrams along $\ell .{ }^{1}$ We do this along each line of the grid, and also solve the global linear-programming instance where the center of the shell is restricted to lie on $H$. Thus, we can solve a two-dimensional instance in $O(n \log n+n / \varepsilon)$ time.

Overall, the recursive algorithm for the subcubes of $\mathcal{C}_{i}$ requires $O\left(\left(n / \varepsilon^{d-2}\right) \log n+\right.$ $n / \varepsilon^{d-1}$ ) time. Thus, solving all the linear programming instances for $\mathcal{C}_{1}, \ldots, \mathcal{C}_{m}$ requires

$$
O\left(\frac{n}{\varepsilon^{d-2}}\left(\log n+\frac{1}{\varepsilon}\right) \log (U+1)\right)
$$

time.

Even though Theorem 3.3 is not fully satisfactory, for all practical purposes the assumptions in the theorem are reasonable. For example, in the plane, if the points in $S$ span an angle of at least $\theta \in[0, \pi / 2]$ with respect to the center $c$ of $\mathcal{A}^{*}(S)$, then $r_{\text {mid }}(c)=O(\Delta / \sin \theta)=O(\Delta / \theta)$. In this case we can compute an annulus that contains $S$ and has width at most $(1+\varepsilon) \omega^{*}(S)$ in time $O\left(\left(n / \varepsilon^{2}\right) \log (1 / \theta)\right)$ or in time $O(n(\log n+1 / \varepsilon) \log (1 / \theta))$.

For $d=2$ the algorithm of Theorem 3.3 can be further simplified and improved, by noting that in this case the power diagrams are (regular) nearest- and furthest-neighbor Voronoi diagrams, and that they need to be computed only once. We thus obtain the following.

\footnotetext{
${ }^{1}$ As a matter of fact, we can compute instead, also in linear time, the minimum difference along $\ell$ between the (unsquared) distances to the farthest and nearest neighbors (which is the width of the minimum-width shell whose center is restricted to lie on $\ell$ ). This optimization may yield a better approximation to the minimum width.
} 
Theorem 3.4. Let $S$ be a set of $n$ points in the plane, $\varepsilon>0$, and $U>0$. One can compute an annulus $\mathcal{A} \supset S$ of width at most $(1+\varepsilon) \omega^{*}(S, U)$ in time $O(n \log n+$ $(n / \varepsilon) \log (U+1))$.

We next modify the algorithm APPROX_SHELL so that it produces in all cases a shell containing $S$ of width at most $(1+\varepsilon) \omega^{*}(S)$.

Lemma 3.5. For $U>6$, we have

$$
\omega^{*}(S, U) \leq \omega^{*}(S)+\frac{8 \cdot \operatorname{diam}(S)}{U} .
$$

Proof. Let $\mathcal{A}^{*}$ be a minimum-width shell containing $S$, with center $p$ and width $\omega^{*}=$ $\omega^{*}(S)$. Put $\Delta=\operatorname{diam}(S)$. It suffices to consider the case $\omega^{*}(S, U) \neq \omega^{*}(S)$, so we have $r_{\text {mid }}(p)>U \Delta$.

Let $\mathcal{V}$ be a circular cone centered at $p$, containing $S$, and having the smallest opening angle. Let $V=\mathcal{V} \cap \mathcal{A}^{*}$. Clearly, $V$ contains $S$. Since $r_{\text {mid }}(p)>6 \Delta, \mathcal{V}$ spans less than a halfspace. Let $v$ be the ray emanating from $p$ along the axis of symmetry of $\mathcal{V}$; see Fig. 5. Let $b$ and $c$ be the points where $v$ meets the inner and outer spheres of $\mathcal{A}^{*}$, respectively. Let $u$ be a point on the segment $p b$ at distance $r=U \Delta / 2$ from $b$. Let $\mathcal{W}$ be the smallest circular cone centered at $u$, with axis of symmetry along $v$ and containing $V$. Let $\sigma$ be the $(d-2)$-sphere formed by intersecting $\partial \mathcal{W}$ with the sphere of radius $r$ centered at $u$, and let $a$ and $l$ denote the center and radius of $\sigma$, respectively (see Fig. 5). Consider the portion of $\mathcal{W}$ lying on the same side as $p$ and $u$ of the hyperplane through $c$ and orthogonal to $v$, and let $R$ denote the maximum distance from $u$ to a point in this portion. The shell $\mathcal{A}^{\prime}$ centered at $u$ with radii $r$ and $R$, encloses $V$ and thus also covers $S$. We now estimate $\omega(u)$ by obtaining an upper bound on the width of $\mathcal{A}^{\prime}$.

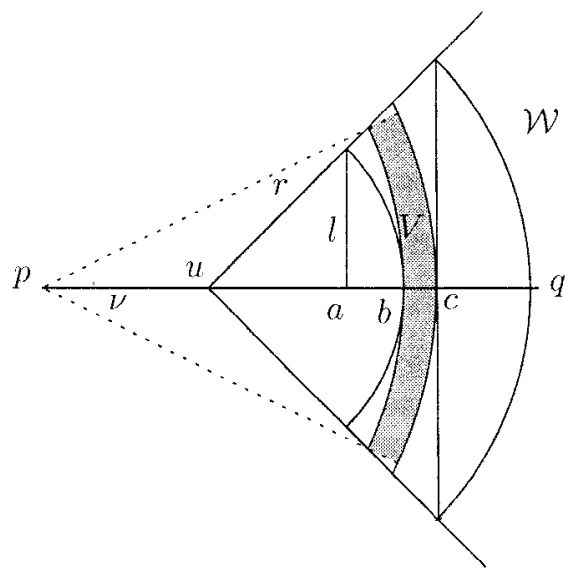

Fig. 5. Construction for the proof of Lemma 3.5. 
Let $q$ be the point on $v$ at distance $R$ from $u$, as shown in Fig. 5. We have $\omega(u) \leq$ $\omega^{*}+d(c, q)$. However, $d(u, a)=\sqrt{r^{2}-l^{2}}$ and

$$
d(a, b)=r-\sqrt{r^{2}-l^{2}}=\frac{l^{2}}{r+\sqrt{r^{2}-l^{2}}} \leq \frac{l^{2}}{r} .
$$

By similarity, we have $d(c, q)=d(a, b)\left(\left(r+\omega^{*}\right) /(d(u, a))\right.$.

We have $\Delta=2 r / U \leq r / 3$. Note that $\omega^{*}<\Delta$ and that $l \leq \Delta$. To see the latter inequality, project $S$ centrally, toward $p$, into the inner sphere of $\mathcal{A}^{*}$. Next, project those points toward $u$, to the sphere $\delta$ of radius $r$ about $u$. The image $\hat{S}$ of $S$ falls inside the projection of $V$ onto $\delta$ which, by construction, is contained in the cap $\delta \cap \mathcal{W}$. Furthermore, any point of $S$ which lies on $\partial \mathcal{V}$, is mapped to a point that lies on $\partial \mathcal{W}$. As can be verified, the segment $p q$ intersects $\mathcal{C H}(\hat{S})$, which implies that $l \leq \Delta(\mathcal{C H}(\hat{S}))$. Since the projection does not increase the distances between points, the diameter of $\hat{S}$ is at most $\Delta$, which implies that $l \leq \Delta$, and

$$
d(u, a)=\sqrt{r^{2}-l^{2}} \geq r \sqrt{1-\frac{1}{9}} \geq \frac{r}{2} .
$$

Hence, we have

$$
d(c, q) \leq \frac{l^{2}}{r} \cdot \frac{2 r}{r / 2}=\frac{4 l^{2}}{r} .
$$

Putting things together,

$$
\begin{aligned}
d(b, q) & =\omega^{*}+d(c, q) \leq \omega^{*}+\frac{4 l^{2}}{r} \leq \omega^{*}+\frac{4 \Delta^{2}}{r} \\
& \leq \omega^{*}+\frac{4 \Delta^{2}}{U \Delta / 2}=\omega^{*}+\frac{8 \Delta}{U} .
\end{aligned}
$$

Note that

$$
\begin{aligned}
r_{\text {mid }}(u) & \leq r+d(b, q)-\frac{\omega^{*}}{2} \leq r+\frac{\omega^{*}}{2}+\frac{8 \Delta}{U}<\frac{3 r}{2}+\frac{8 \Delta}{U} \\
& =\Delta\left(\frac{3 U}{4}+\frac{8}{U}\right)<U \cdot \Delta .
\end{aligned}
$$

Hence $\omega^{*}(S, U) \leq w(u) \leq \omega^{*}+8 \Delta / U$, as asserted.

Corollary 3.6. Let $\varepsilon>0, U>6$ be two positive constants. One can compute, in time $O\left(\left(\left(n / \varepsilon^{d-2}\right) \log n+n / \varepsilon^{d-1}\right) \log (U+1)\right)$ or $O\left(n / \varepsilon^{d} \log (U+1)\right)$, a shell of width at most

$$
(1+\varepsilon)\left[\omega^{*}(S)+\frac{8 \Delta}{U}\right]
$$

that contains $S$, where $\Delta=\operatorname{diam}(S)$.

Finally, we describe the general approximation algorithm. Let APPROX_DiAm $(S)$ be the procedure that computes in linear time a $\sqrt{3}$-approximation $\Delta_{0}$ of $\Delta(S)=\operatorname{diam}(S)$ 
Algorithm APPROX_SHELL_2 $(S, \varepsilon)$

$$
\begin{aligned}
& \omega=\Delta_{0}=\text { APPROX_DiAM }(S) ; \quad \omega_{\text {old }}=\infty ; \\
& \text { while } \omega<\omega_{\text {old }} / 2 \text { do } \\
& \qquad U=\frac{50 \sqrt{3} \Delta_{0}}{\varepsilon} \cdot \frac{1}{\omega} ; \\
& \qquad \mathcal{A}(p)=\operatorname{APPROX} \operatorname{SHELL}(S, U, \varepsilon / 8) ; \\
& \omega_{\text {old }}=\omega ; \quad \omega=\omega(p) ; \\
& \text { end while } \\
& \text { return } \mathcal{A}(p) ;
\end{aligned}
$$

Fig. 6. The $\varepsilon$-approximation algorithm APPROX_SHELL_2 for arbitrary dimensions.

(see [13] or the discussion at the beginning of the proof of Lemma 3.2). The approximation algorithm is depicted in Fig. 6.

Theorem 3.7. Given a set $S$ of $n$ points in $\mathbb{R}^{d}$ and a parameter $0<\varepsilon<1$, APPROX_SHELL_2 computes a shell of width at most $(1+\varepsilon) \omega^{*}(S)$. With an appropriate optimization of the calls to APPROX_SHELL, the running time is either

$$
O\left(\frac{n}{\varepsilon^{d}} \log \left(\frac{\Delta}{\omega^{*}(S) \varepsilon}\right)\right) \quad \text { or } \quad O\left(\frac{n}{\varepsilon^{d-2}}\left(\log n+\frac{1}{\varepsilon}\right) \log \left(\frac{\Delta}{\omega^{*}(S) \varepsilon}\right)\right) .
$$

Proof. If $\omega^{*}(S)=0$, the algorithm terminates after the first iteration. Otherwise, it eventually terminates, as the positive width returned in each call decreases by at least a factor of two, but is no smaller than the optimum width $\omega^{*}(S)$.

Suppose the while loop is executed $m$ times. Let $\omega_{i}, U_{i}$ be the values of $\omega$ and $U$ computed in the $i$ th iteration of the loop. Then, putting $\omega^{*}=\omega^{*}(S)$,

$$
\begin{aligned}
\omega_{m} & \leq\left(1+\frac{\varepsilon}{8}\right) \omega^{*}+\left(1+\frac{\varepsilon}{8}\right) \frac{8 \Delta}{U_{m}} \\
& \leq\left(1+\frac{\varepsilon}{8}\right) \omega^{*}+\left(1+\frac{\varepsilon}{8}\right) \frac{8 \Delta}{50 \sqrt{3} \Delta_{0} /\left(\omega_{m-1} \varepsilon\right)} \\
& \leq\left(1+\frac{\varepsilon}{8}\right) \omega^{*}+\left(1+\frac{\varepsilon}{8}\right) \frac{4 \varepsilon \omega_{m-1}}{25} \\
& \leq\left(1+\frac{\varepsilon}{8}\right) \omega^{*}+\frac{9 \varepsilon \omega_{m}}{25},
\end{aligned}
$$

by Lemma 3.5 , and since $w_{m} \geq w_{m-1} / 2$. Thus,

$$
\omega_{m} \leq \frac{1+\varepsilon / 8}{1-9 \varepsilon / 25} \omega^{*} \leq(1+\varepsilon) \omega^{*} .
$$

Note that for all $i<m$, we have $\omega_{i}<\Delta_{0} \sqrt{3} / 2^{i}$. Hence, $\omega^{*} \leq \omega_{m-1} \leq \Delta_{0} \sqrt{3} / 2^{m-1}$, implying that $m=O\left(\log \left(\Delta / \omega^{*}\right)\right)$ and $U_{m}=O\left(\Delta /\left(\omega^{*} \varepsilon\right)\right)$.

Note that the $i$ th call to APPROX_SHELL (executed, say, by the first algorithm of Theorem 3.3) constructs a tiling of $\mathfrak{C}_{i}=C\left(o,\left(2 U_{i}+2\right) \Delta\right)$, and computes $\mathcal{E}(S, C)$ for each cube $C$ in this tiling. By modifying the algorithm so that it computes $\mathcal{E}(S, C)$ only for the new cubes $C$ in the tiling (that is, ignoring cubes that are covered by cubes 
produced in earlier iterations), it follows that the running time of the $i$ th iteration can be improved to $O\left(n / \varepsilon^{d}\right)\left(1+\log \left(\left(U_{i}+1\right) /\left(U_{i-1}+1\right)\right)\right)$, for $i=2, \ldots, m$. Overall, the running time of the algorithm is thus

$$
\begin{gathered}
O\left(\frac{n}{\varepsilon^{d}} \log U_{1}+1+\sum_{i=2}^{m} \frac{n}{\varepsilon^{d}}\left(1+\log \frac{U_{i}+1}{U_{i-1}+1}\right)\right) \\
=O\left(\frac{n}{\varepsilon^{d}}\left(m+\log \left(U_{m}+1\right)\right)\right)=O\left(\frac{n}{\varepsilon^{d}} \log \frac{\Delta}{\omega^{*} \varepsilon}\right) .
\end{gathered}
$$

The other time bound follows if we execute APPROX_SHELL using the second algorithm of Theorem 3.3.

Remark 3.8. The running times of the results of this section can be improved by using a data structure for linear-programming queries. Since these data structures are quite complicated, the resulting improvements are only of theoretical interest and are thus omitted. See [7] for more details.

\section{Approximation Algorithms in the Plane}

Let $S$ be a set of $n$ points in the plane. We first present an $O(n \log n)$-time algorithm that computes an annulus containing $S$ whose width is at most $2 \omega^{*}$. We then describe an algorithm that, given a parameter $\varepsilon>0$, computes in $O\left(n \log n+n / \varepsilon^{2}\right)$ time an annulus containing $S$ whose width is at most $(1+\varepsilon) \omega^{*}$.

\subsection{A 2-Approximation Algorithm}

We first compute the width width $(S)$ of $S$ (i.e., the minimum distance between a pair of parallel lines that contain $S$ between them). Next, we compute a diametral pair of $S$, i.e., a pair $p, q \in S$ such that $d(p, q)=\operatorname{diam}(S)$. Both of these steps take $O(n \log n)$ time. Let $\ell$ be the perpendicular bisector of $p q$. We compute $\operatorname{Vor}_{\mathrm{N}}(S, \ell)$ and $\operatorname{Vor}_{\mathrm{F}}(S, \ell)$, merge the vertices of the two diagrams into a single sorted list $V$, and compute the point $v^{*}$ that minimizes $\omega(v)$ over all $v \in \ell$. The latter stages can be done in $O(|V|)$ time because, between any pair of successive points of $V, \omega(v)$ coincides with the difference of distances to two fixed points of $S$. If width $(S) \geq \omega\left(v^{*}\right)$, we return $\mathcal{A}\left(v^{*}\right)$; otherwise, we return a strip of width width $(S)$ that contains $S$. The algorithm obviously returns a (proper or degenerate) annulus that contains $S$, and it runs in $O(n \log n)$ time.

Theorem 4.1. The width of the annulus computed by the above algorithm is at most $2 \omega^{*}$. That is,

$$
\min \left\{\omega\left(v^{*}\right), \operatorname{width}(S)\right\} \leq 2 \omega^{*} .
$$




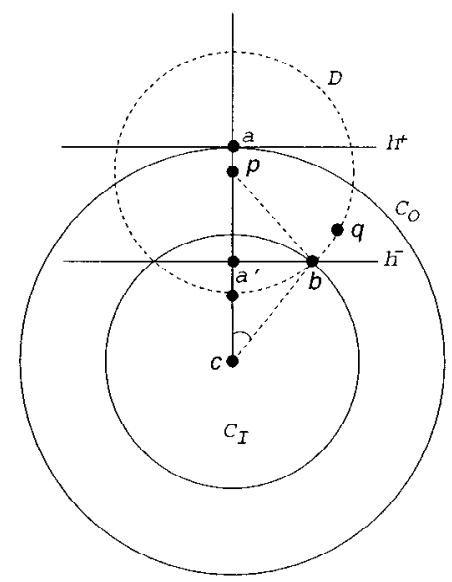

Fig. 7. The minimum-width annulus and the strip defined by $h^{-}, h^{+}$.

Remark 4.2. An easy calculation, based on area considerations and using the fact that $p q$ is a diameter, shows that $S$ can be covered by a strip of width at most $2 \operatorname{width}(S)$ and bounding lines parallel to $p q$. Therefore, $\omega\left(v^{*}\right) \leq 2$ width $(S)$, which, in view of Theorem 4.1 , implies that $\omega\left(v^{*}\right) \leq 4 \omega^{*}$, so that skipping the width computation in the algorithm gives a 4-approximation of $\omega^{*}$.

Let $\Delta=\operatorname{diam}(S)$. Let $C_{\mathrm{O}}$ and $C_{\mathrm{I}}$ be the outer and inner circles of an annulus $\mathcal{A}^{*}$ of width $\omega^{*}$ that contains $S$, and let $c$ be the center of $\mathcal{A}^{*}$ (we can clearly assume that $c$ is not at infinity). Let $p, q$ be the diametral pair computed by the algorithm. Without loss of generality, we can assume that $c$ is the origin, $p=(0,1), 1=d(c, p) \geq$ $d(c, q)$, and $x(q) \geq 0$ (see Fig. 7). Let $D$ be the circle of radius $d(p, q)=\Delta$ centered at $p$.

Lemma 4.3. If $\Delta \leq 1$, then $S$ is contained in a horizontal strip of width at most $\omega^{*}+\Delta^{2} / 2$.

Proof. Let $a$ be the topmost point of $C_{\mathrm{O}}$. Since $\Delta \leq 1, c \notin \operatorname{int}(D)$, which implies that either $D$ lies fully above $C_{\mathrm{I}}$ (i.e., the horizontal line passing through the topmost point of $C_{\mathrm{I}}$ strictly separates $D$ and $C_{\mathrm{I}}$ ) or $\partial D$ and $C_{\mathrm{I}}$ intersect at two points with positive $y$-coordinates; the case in which $\partial D$ and $C_{\mathrm{I}}$ touch can be handled by essentially the same argument. The first situation is impossible: since $S \subseteq D$, we can grow $C_{\mathrm{I}}$ and still have $S$ lie in the shrunken annulus, contrary to the minimality of $\mathcal{A}^{*}$. Let $b$ be the intersection point of $\partial D$ and $C_{\mathrm{I}}$ lying to the right of the $y$-axis. Let $h^{-}, h^{+}$be the horizontal lines passing through $b$ and $a$, respectively. Since $S \subseteq \mathcal{A}^{*} \cap D$, the strip bounded by $h^{-}, h^{+}$ contains $S$; see Fig. 7. Let $a^{\prime}$ be the intersection point of $h^{-}$and the $y$-axis. Then, using 
the law of cosines,

$$
\begin{aligned}
d\left(a^{\prime}, c\right) & =d(c, b) \cos (\measuredangle b c p) \\
& =d(c, b) \frac{d(p, c)^{2}+d(c, b)^{2}-d(p, b)^{2}}{2 d(p, c) d(c, b)} \\
& =\frac{1+r_{\mathrm{I}}^{2}-\Delta^{2}}{2},
\end{aligned}
$$

where $r_{\mathrm{I}}$ is the radius of $C_{\mathrm{I}}$. Therefore the width of the strip is

$$
\begin{aligned}
d(a, c)-d\left(a^{\prime}, c\right) & =r_{\mathrm{I}}+\omega^{*}-\frac{1+r_{\mathrm{I}}^{2}-\Delta^{2}}{2} \\
& =\omega^{*}+\frac{\Delta^{2}}{2}-\frac{\left(1-r_{\mathrm{I}}\right)^{2}}{2} \leq \omega^{*}+\frac{\Delta^{2}}{2} .
\end{aligned}
$$

Hence, if $\Delta \leq 1$ and $\omega^{*} \geq \Delta^{2} / 2$, the algorithm computes an annulus (that is, a strip) of width at most $2 \omega^{*}$. We now assume that either $\Delta>1$ or $\omega^{*}<\Delta^{2} / 2$.

Let $C_{p q}$ be the circle that passes through $p$ and $q$ and whose center $\xi$ lies on the $y$-axis; see Fig. 8. We will show that all points of $S$ lie within distance $\omega^{*}$ from $C_{p q}$, which implies that the annulus centered at $\xi$ with the inner radius $d(\xi, p)-\omega^{*}$ and the outer radius $d(\xi, p)+\omega^{*}$ contains $S$. Since $\xi$ lies on the perpendicular bisector of $p q$, the thinnest annulus that the algorithm computes is certainly no wider than $\mathcal{A}(\xi)$, i.e., its width is at most $2 \omega^{*}$.

Since $d(c, p) \geq d(c, q), C_{p q}$ lies inside the circle passing through $p$ and centered at $c$, and therefore it also lies inside $C_{\mathrm{O}}$. However, $C_{p q}$ may intersect $C_{\mathrm{I}}$ (as in Fig. 8). Let $\Gamma \subseteq C_{p q}$ be the circular arc from $p$ to $q$ in the clockwise direction. A simple calculation shows that the distance from $c$ to the points of $\Gamma$ decreases monotonically along $\Gamma$. Since $p, q \in \mathcal{A}^{*}$, the entire arc $\Gamma$ lies inside $\mathcal{A}^{*}$.

Lemma 4.4. If $\Delta>1$ or $\omega^{*}<\Delta^{2} / 2$, then $\measuredangle p q c<\pi / 2$.

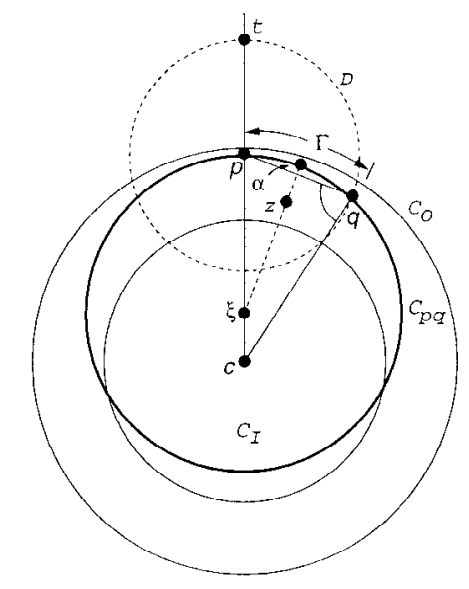

Fig. 8. The minimum-width annulus and the circle $C_{p q}$. 
Proof. If $\Delta>1$, then $c \in \operatorname{int}(D)$. We then have $\measuredangle p q c<\measuredangle p q m<\measuredangle t q m=\pi / 2$, where $m$ is the bottommost point of $D$. Next, assume that $\omega^{*}<\Delta^{2} / 2$. Since $d(c, p)=1$, $d(p, q)=\Delta$, and $1 \geq d(c, q) \geq 1-\omega^{*}$, we obtain

$$
\begin{aligned}
\cos (\measuredangle p q c) & =\frac{d(p, q)^{2}+d(c, q)^{2}-d(c, p)^{2}}{2 d(p, q) d(c, q)} \\
& =\frac{\Delta^{2}+d(c, q)^{2}-1}{2 \Delta d(c, q)} \\
& \geq \frac{\Delta^{2}+\left(1-\omega^{*}\right)^{2}-1}{2 \Delta} \\
& =\frac{\Delta^{2}-2 \omega^{*}+\omega^{* 2}}{2 \Delta} \\
& >0 .
\end{aligned}
$$

The last inequality follows from the assumption that $\omega^{*}<\Delta^{2} / 2$. This completes the proof of the lemma.

We now prove that, for any point $z \in S$, the distance $d\left(z, C_{p q}\right)$ between $C_{p q}$ and $z$ is at most $\omega^{*}$. We will prove the claim for points with positive $x$-coordinates; the same argument applies to points with negative $x$-coordinates. Let $\alpha$ be the intersection point of $C_{p q}$ with the ray emanating from $\xi$ in direction $\vec{\xi} z$; see Fig. 8. Then $d\left(z, C_{p q}\right)=d(z, \alpha)$.

If $z \in \operatorname{int}\left(C_{p q}\right)$, then let $\beta$ be the intersection point of $C_{p q}$ with the ray emanating from $z$ in direction $\overrightarrow{c z}$ (see Fig. 9); otherwise, let $\beta$ be the intersection point of $C_{p q}$ with the ray emanating from $z$ in direction $\overrightarrow{z c}$. The point $\beta$ exists since $c$ lies inside $C_{p q}$, as $\measuredangle p q c<\pi / 2$. Since $\alpha$ lies on the line passing through $z$ and the center of $C_{p q}$, i.e., $\alpha$ is the nearest point on $C_{p q}$ from $z, d(z, \alpha) \leq d(z, \beta)$.

Lemma 4.5. $d(z, \beta)<\omega^{*}$.

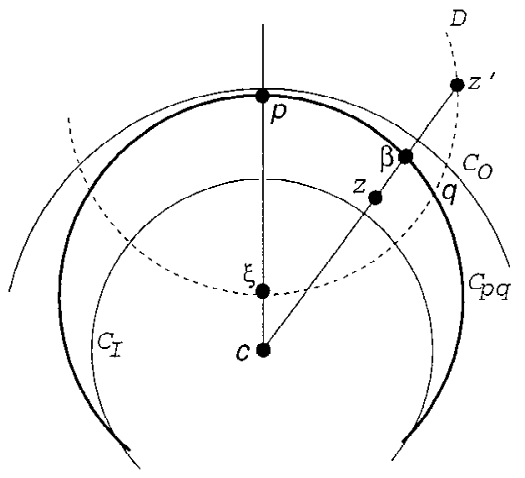

(i)

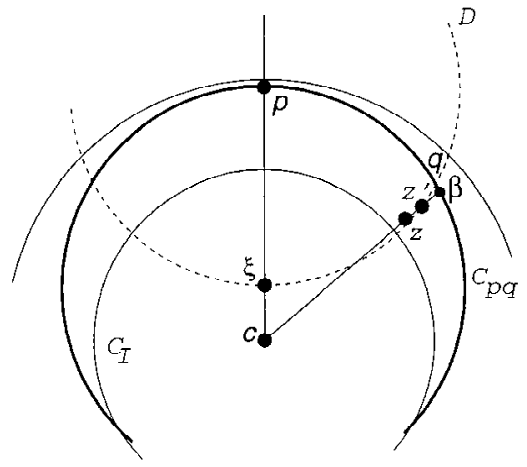

(ii)

Fig. 9. Illustration of the proof of Lemma 4.5. (i) $z^{\prime} \in D[a, q]$, (ii) $z^{\prime} \notin D[a, q]$. 
Proof. We will prove that $\beta$ lies in the annulus $\mathcal{A}^{*}$. Let $z^{\prime}$ be the intersection point of $D$ with the ray $\overrightarrow{c z}$, such that $z$ lies between $c$ and $z^{\prime}$. For two points $x, y \in D$, let $D[x, y] \subseteq D$ denote the circular arc from $x$ to $y$ in the clockwise direction. Let $t$ be the topmost point of $D$. There are two cases to consider:

Case (i): $z^{\prime} \in D[t, q]$. By Lemma 4.4, $\measuredangle p q c<\pi / 2$, therefore $D[t, q]$ lies in the wedge formed by the positive $y$-axis and the ray $\overrightarrow{c q}$. This in turn implies that $\beta \in \Gamma$ irrespective of whether $z$ lies inside or outside $C_{p q}$; see Fig. 9(i). As noted earlier, $\Gamma \subset \mathcal{A}^{*}$, so $\beta \in \mathcal{A}^{*}$, as claimed.

Case (ii): $z^{\prime} \notin D[t, q]$. Note that $q$ is an intersection point of circles $D$ and $C_{p q}$ and their second point of intersection is the mirror image of $q$ on the other side of the $y$-axis. Therefore the portion of $D$ from $q$ to its bottommost point in the clockwise direction lies inside $C_{p q}$. Since $z^{\prime}$ has a positive $x$-coordinate and $z^{\prime} \notin D[t, q], z^{\prime}$ lies on the portion of $D$ inside $C_{p q}$. Therefore $\beta$ lies after $z^{\prime}$ on the ray $\overrightarrow{c z}$ (see Fig. 9(ii)) and

$$
r_{\mathrm{I}} \leq d(c, z) \leq d\left(c, z^{\prime}\right)<d(c, \beta)<r_{\mathrm{O}},
$$

where the last inequality follows from the fact that $C_{p q} \subset \operatorname{int}\left(C_{\mathrm{O}}\right)$. This implies that $\beta \in \mathcal{A}^{*}$, as desired.

We thus have $d(z, \beta)<\omega^{*}$.

Lemmas 4.3 and 4.5 imply the theorem.

\subsection{A $(1+\varepsilon)$-Approximation Algorithm}

In this subsection we present a $(1+\varepsilon)$-approximation algorithm for the (planar) minimumwidth annulus problem. The algorithm is a combination of the approximation techniques developed above, and is described in Fig. 10.

Theorem 4.6. The width of the annulus output by PLANAR_APPROX_SHELL $(S, \varepsilon)$ is at most $(1+\varepsilon) \omega^{*}(S)$, and the running time of the algorithm is $O\left(n \log n+n / \varepsilon^{2}\right)$.

Proof. If $r_{\text {mid }}\left(\mathcal{A}^{*}(S)\right) \leq U \Delta(S)$, the correctness and the bound on the running time are consequences of the previous algorithms, so assume that $r_{\text {mid }}\left(\mathcal{A}^{*}(S)\right)>U \Delta(S)$. Let $C^{*}$ be the middle circle of $\mathcal{A}^{*}(S)$, and let $c^{*}, r^{*}$ denote the center and the radius of $C^{*}$, respectively. Without loss of generality, assume that $c^{*}$ lies (far away) below the $x$-axis. Let $I_{p}$ and $I_{q}$ denote the vertical segments spanned by the points of $P_{p}$ and of $P_{q}$, respectively.

We have that $\omega^{*}(S)<\Delta(S) / 300$ (otherwise, by Lemma $3.5, \mathcal{A}^{\prime \prime}$ is the required approximation), which implies that both $I_{p}$ and $I_{q}$ are "short" compared with the diameter of $S$. Moreover, the radius of the optimal solution is huge (i.e., at least $(10,000 / \varepsilon) \Delta(S))$; namely, the sector of the optimal annulus that contains $S$ spans a very small angle.

Using the fact that $S$ lies in a small sector of the annulus, it can be shown that $C^{*}$ crosses both $I_{p}$ and $I_{q}$, at two respective points $u, v$. Let $u_{1}$ (resp. $v_{1}$ ) denote the point of $P_{p}$ (resp. of $P_{q}$ ) that lies immediately below $u$ (resp. $v$ ). We first translate $C^{*}$ downward, 
Algorithm PLANAR_APPROX_SHELL $(S, \varepsilon)$

1. Run the 2-approximation algorithm of Theorem 4.1. Let $\mathcal{A}^{\prime}$ be the resulting annulus. If the width $\omega^{\prime}$ of $\mathcal{A}^{\prime}$ is 0 , then return $\mathcal{A}^{\prime}$.

2. Compute the farthest- and nearest-neighbor Voronoi diagrams $\operatorname{Vor}_{\mathrm{F}}(S), \operatorname{Vor}_{\mathrm{N}}(S)$.

3. Set $U=10000 / \varepsilon$. Compute an annulus $\mathcal{A}^{\prime \prime}$ of width $\leq(1+\varepsilon / 2) \omega^{*}(S, U)$, using the algorithm of Theorem 3.4. (Either $\mathcal{A}^{\prime \prime}$ is the required $\varepsilon$-approximation, or $r_{\text {mid }}\left(\mathcal{A}^{*}(S)\right)>U \Delta(S)$.)

4. Compute a pair of points $p, q \in S$ that realize the diameter of $S$. We assume without loss of generality that $p=(-1,0), q=(1,0)$. Set $\delta=\varepsilon \omega^{\prime} / 20$, let $P_{p}=P(p, \delta, \varepsilon), P_{q}=P(q, \delta, \varepsilon)$, where

$$
P(z, \delta, \varepsilon)=\{z+(0, \delta) i \mid i=-\lceil 40 / \varepsilon\rceil, \ldots,\lceil 40 / \varepsilon\rceil\} .
$$

See Fig. 11.

5. For each pair $u \in P_{p}, v \in P_{q}$ compute the minimum-width annulus whose center lies on the perpendicular bisector of $u v$.

6. Output the minimum-width annulus among those computed.

Fig. 10. A strongly polynomial $\varepsilon$-approximation algorithm PLANAR_APPROX_SHELL for the planar case

till it first hits either $u_{1}$ or $v_{1}$. Suppose, without loss of generality, that it first hits $v_{1}$. Let $C$ denote the translated circle. Clearly, the center $c$ of $C$ lies vertically below $c^{*}$ at distance less than $\delta$. In particular, for any $s \in S$ we have $\left|d(c, s)-d\left(c^{*}, s\right)\right| \leq d\left(c, c^{*}\right)<\delta$. Put $D(C, S)=\max _{s \in S} d(C, s)$, and $\omega=2 D(C, S)$ and observe that

$$
\omega<2\left(D\left(C^{*}, S\right)+\delta\right)=\omega^{*}+2 \delta \leq\left(\frac{1+\varepsilon}{5}\right) \omega^{*} .
$$

Next, shrink $C$ by moving its center from $c$ toward $v_{1}$ while keeping $v_{1}$ on the circle, until it also passes through $u_{1}$. Let $C^{\prime}$ denote the new circle and let $c^{\prime}$ denote its center. See Fig. 11.

The distance from $c$ to points on $C^{\prime}$ decreases monotonically as we traverse $C^{\prime}$ from $v_{1}$ counterclockwise until we reach the point on $C^{\prime}$ antipodal to $v_{1}$. Let $s$ be any point of $S$. The ray $\rho$ from $c$ toward $s$ crosses $C$ at a point $w$ and $C^{\prime}$ at a point $w^{\prime}$. We have $d\left(w^{\prime}, s\right) \leq d(w, s)+d\left(w, w^{\prime}\right) \leq \omega / 2+d\left(w, w^{\prime}\right)$. Again, using the fact that $S$ lies in

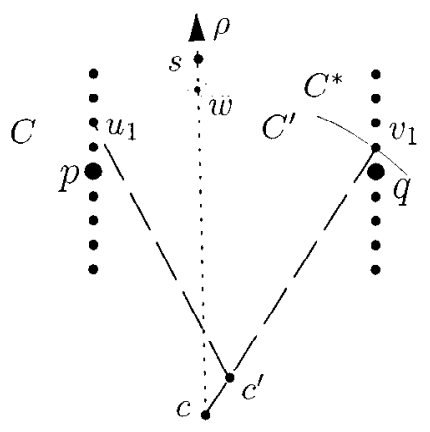

Fig. 11. Proof of correctness of PLANAR_APPROX_SHELL. 
a small sector of the annulus $\mathcal{A}^{*}(S)$, one can argue that $d\left(w, w^{\prime}\right)$ attains its maximum when $w^{\prime}$ is near $u_{1}$, and this maximum is smaller than $2 \delta$ (the latter statement is easy to verify, using the fact that the line through $w$ and $w^{\prime}$ is almost vertical). (Note that there is no need to apply this reasoning to the portion of $C^{\prime}$ clockwise from $v_{1}$, since no point of $S$ lies to the right of $v_{1}$.) This implies that

$$
\omega\left(c^{\prime}\right) \leq 2 D\left(C^{\prime}, S\right) \leq \omega+2 \delta \leq\left(1+\frac{2 \varepsilon}{5}\right) \omega^{*} \leq(1+\varepsilon) \omega^{*} .
$$

Since $c^{\prime}$ lies on the perpendicular bisector of $u_{1} v_{1}$, it follows that the width of the annulus output by the algorithm is at most $\omega\left(c^{\prime}\right)<(1+\varepsilon) \omega^{*}$, as asserted. Step 2 takes $O(n \log n)$ time. By Theorem 3.4, Step 3 takes $O(n \log n+n / \varepsilon)$ time. A diametrical pair of $S$ can be computed in $O(n \log n)$ time. Finally, for each pair $(u, v) \in P_{p} \times P_{q}$, Step 5 takes $O(n)$ time. Hence, the total running time is $O\left(n \log n+n / \varepsilon^{2}\right)$.

\section{References}

1. P. K. Agarwal, B. Aronov, S. Har-Peled, and M. Sharir, Approximation and exact algorithms for minimumwidth annuli and shells, Proc. 15th Annu. ACM Sympos. Comput. Geom., 1999, pages 380-389.

2. P. K. Agarwal, B. Aronov, and M. Sharir, Computing envelopes in four dimensions with applications, SIAM J. Comput., 26 (1997), 1714-1732.

3. P. K. Agarwal and M. Sharir, Efficient randomized algorithms for some geometric optimization problems, Discrete Comput. Geom., 16 (1996), 317-337.

4. P. K. Agarwal, M. Sharir, and S. Toledo, Applications of parametric searching in geometric optimization, J. Algorithms, 17 (1994), 292-318.

5. A. Aggarwal, L. J. Guibas, J. Saxe, and P. W. Shor, A linear-time algorithm for computing the Voronoi diagram of a convex polygon, Discrete Comput. Geom., 4 (1989), 591-604.

6. F. Aurenhammer and R. Klein, Voronoi diagrams, in Handbook of Computational Geometry (J.-R. Sack and J. Urrutia, eds.), Elsevier Science/North-Holland, Amsterdam, 1999.

7. T. M. Chan, Approximating the diameter, width, smallest enclosing cylinder, and minimum-width annulus, Proc. 16th Annu. ACM Sympos. Comput. Geom., 2000, pages 300-309.

8. M. de Berg, J. Bose, D. Bremner, S. Ramaswami, and G. Wilfong, Computing constrained minimum-width annuli of point sets, Proc. 5th Workshop Algorithms Data Struct., Lecture Notes in Computer Science, Vol. 1272, Springer-Verlag, Berlin, 1997, pp. 3-16.

9. C. A. Duncan, M. T. Goodrich, and E. A. Ramos, Efficient approximation and optimization algorithms for computational metrology, Proc. 8th ACM-SIAM Sympos. Discrete Algorithms, 1997, pp. 121-130.

10. M. Dyer and N. Megiddo, Linear programming in low dimensions, in Handbook of Discrete and Computational Geometry (J. E. Goodman and J. O’Rourke, eds.), CRC Press, Boca Raton, FL, 1997, pp. 699-710.

11. H. Ebara, N. Fukuyama, H. Nakano, and Y. Nakanishi, Roundness algorithms using the Voronoi diagrams, Abstracts 1st Canad. Conf. Comput. Geom., 1989, p. 41.

12. H. Edelsbrunner, L. J. Guibas, and J. Stolfi, Optimal point location in a monotone subdivision, SIAM J. Comput., 15 (1986), 317-340.

13. O. Egecioglu and B. Kalantari, Approximating the diameter of a set of points in the Euclidean space, Inform. Process. Lett., 32 (1989), 205-211.

14. L. W. Foster, GEO-METRICS II: The Application of Geometric Tolerancing Techniques, Addison-Wesley, Reading, MA, 1982.

15. J. García-Lopez and P. Ramos, Fitting a set of points by a circle, Proc. 13th Annu. ACM Sympos. Comput. Geom., 1997, pp. 139-146.

16. S. Har-Peled. Constructing approximate shortest path maps in three dimensions, SIAM J. Comput., 28 (1999), 1182-1197.

17. R. Kumar and D. Sivakumar, Roundness estimation via random sampling, Proc. 10th ACM-SIAM Sympos. Discrete Algorithms, 1999, pp. 603-612. 
18. V. B. Le and D. T. Lee, Out-of-roundness problem revisited, IEEE Trans. Pattern Anal. Mach. Intell., 13 (1991), 217-223.

19. J. Matoušek, M. Sharir, and E. Welzl, A subexponential bound for linear programming, Algorithmica, 16 (1996), 498-516.

20. K. Mehlhorn, T. Shermer, and C. Yap, A complete roundness classification procedure, Proc. 13th Annu. ACM Sympos. Comput. Geom., 1997, pp. 129-138.

21. J. S. B. Mitchell, D. M. Mount, and C. H. Papadimitriou, The discrete geodesic problem, SIAM J. Comput., 16 (1987), 647-668.

22. F. P. Preparata and M. I. Shamos, Computational Geometry: An Introduction, Springer-Verlag, New York, 1985.

23. T. J. Rivlin, Approximating by circles, Computing, 21 (1979), 93-104.

24. U. Roy, C. R. Liu, and T. C. Woo, Review of dimensioning and tolerancing: representation and processing, Comput. Aided Design, 23 (1991), 466-483.

25. U. Roy and X. Zhang, Establishment of a pair of concentric circles with the minimum radial separation for assessing roundness error, Comput. Aided Design, 24 (1992), 161-168.

26. T. C. Shermer and C. K. Yap, Probing for near centers and relative roundness, Proc. ASME Workshop on Tolerancing and Metrology, 1995.

27. M. Smid and R. Janardan, On the width and roundness of a set of points in the plane. Internat. J. Comput. Geom. Appl., 9 (1999), 97-108.

28. K. Swanson, D. T. Lee, and V. L. Wu, An optimal algorithm for roundness determination on convex polygons, Comput. Geom. Theory Appl., 5 (1995), 225-235.

29. C. K. Yap and E.-C. Chang, Issues in the metrology of geometric tolerancing, in Algorithms for Robotic Motion and Manipulation (J.-P. Laumond and M. Overmars, ed.), A.K. Peters, Wellesley, MA, 1997, pp. 393-400.

Received July 6, 1999, and in revised form April 17, 2000. Online publication August 21, 2000. 